\title{
Effets du tabagisme et du niveau de dépendance nicotinique sur les métabolismes anaérobies des footballeurs
}

\author{
Bernard PACKA TCHISSAMBOU ${ }^{1 *}$, Alphonse MASSAMBA ${ }^{1,2}$, \\ Jean Robert MABIALA BABELA ${ }^{3}$ et Prosper SENGA ${ }^{3}$ \\ ${ }^{1}$ Laboratoire de Physiologie de l'Effort et de Biomécanique, Institut Supérieur des Sciences de l'Education \\ Physique et du Sport, Université Marien NGOUABI, Brazzaville (Congo) \\ ${ }^{2}$ Laboratoire de Nutrition, Santé et Motricité Humaine, Institut Supérieur des Sciences de l'Education \\ Physique et du Sport, Université Marien NGOUABI, Brazzaville (Congo) \\ ${ }^{3}$ Département de Médecine, Faculté des Sciences de la Santé, Université Marien NGOUABI, Brazzaville \\ (Congo) \\ *Auteur correspondant ; E-mail: patchiber@yahoo.com
}

\section{RESUME}

Evaluer les effets du tabagisme et de la dépendance nicotinique sur le niveau de développement des métabolismes anaérobies alactique et lactique chez des footballeurs fumeurs. Soixante trois footballeurs non-fumeurs et 63 fumeurs de première division ligue, âgés de 18 à 33 ans, ont été soumis à deux tests d'évaluation de puissance anaérobie maximale alactique (test de Sargent) et lactique (test de Wingate). Des mesures de la lactatémie ont été également effectuées avant le test de Wingate, en fin d'effort, et à 10 minutes de la récupération. Comparés aux nonfumeurs, les fumeurs présentaient une limitation significative $(p<0,001)$ des puissances anaérobies maximales absolues alactique et lactique. Par contre, si les valeurs des puissances anaérobies maximales alactique rapportées au poids et au volume musculaire des fumeurs étaient significativement $(\mathrm{p}<0,01)$ inférieures à celles des non fumeurs. Par contre, celles des puissances anaérobies maximales lactiques étaient non significatives. Par ailleurs, les concentrations plasmatiques de lactates chez les fumeurs étaient plus élevées $(\mathrm{p}<0,001)$ que chez les non-fumeurs. Le niveau de dépendance tabagique a une incidence significative $(\mathrm{p}<0,001)$ sur les différentes variables étudiées. L'inhalation de la fumée de tabac entraîne une baisse de la puissance des métabolismes anaérobies alactique et lactique chez les footballeurs fumeurs, en rapport avec le degré de dépendance nicotinique. Les différences observées entre fumeurs et non fumeurs sont imputables aux modifications des activités enzymatiques au niveau musculaire.

(ㅇ) 2010 International Formulae Group. All rights reserved.

Mots clés: Football, tabagisme, dépendance nicotinique, métabolisme anaérobie, lactatémie.

\section{INTRODUCTION}

Plus de 300 sportifs ont été interrogés en février 2009 à Brazzaville et Pointe-Noire
(Congo), dans le cadre d'un sondage réalisé par le Comité Congolais de Lutte contre le Tabagisme et l'Institut Supérieur des Sciences 
de l'Education Physique et du Sport. Cette enquête a permis de mettre en évidence que $37 \%$ des sportifs se déclarent fumeurs (Packa et Massamba, 2009). Or il est communément admis que le tabac a des effets délétères sur la fonction cardiorespiratoire : réduction des échanges gazeux au niveau des poumons, et diminution du pouvoir oxyphorique du sang (Packa et al., 2004 ; Packa et al., 2001). De plus, les troubles du métabolisme musculaire liés au tabagisme sont importants : augmentation de la lactacidémie musculaire et sanguine, altérations musculaires pures (Longo, 2006 ; Packa, 2003). Ces différents effets concourent donc à diminuer la performance sportive. Toutefois, l'importance physiologique exacte de la limitation induite chez le sujet entraîné, d'une qualité physique à l'autre, reste encore à définir. Ainsi, peu d'études, toutes européennes et américaines, ont porté sur l'influence du niveau de dépendance nicotinique sur les puissances anaérobies alactique et lactique (Lanston et al., 2006 ; Wagner et al., 2000). Or, plusieurs auteurs ont montré que l'homme noir dispose d'une structure histochimique spécifique des muscles des membres inférieurs (Batra et al., 2003 ; Perusse, 2001). Toutefois, à notre connaissance, aucune de ces études n'a concerné les mélano-africains. C'est dans ce but que nous avons entrepris ce travail chez les footballeurs congolais fumeurs. Tenant compte des effets délétères du tabagisme sur l'aptitude physique chez les sujets entraînés, il n'est pas erroné de prédire que la consommation du tabac altère davantage la puissance musculaire chez ces derniers. Les objectifs de cette étude sont donc d'une part d'apprécier l'impact du tabagisme et du niveau de la dépendance nicotinique sur les métabolismes anaérobies alactique et lactique, et d'autre part d'évaluer le niveau de production de l'acide lactique à l'effort et au décours de l'exercice chez les footballeurs fumeurs.

\section{MATERIEL ET METHODES}

L'étude, transversale et de type castémoins, a été menée à Brazzaville et Pointe-
Noire, les deux principales villes du Congo (Brazzaville). Au total, 126 footballeurs de première division des ligues de Brazzaville et de Pointe-Noire (Congo-Brazzaville), ont participé à cette expérimentation. Les sujets, âgés de 18 à 33 ans (moyenne 24,8 $\pm 5,1$ ans) pratiquaient le football de compétition depuis 4 à 9 ans. Ils s'entraînaient quotidiennement pendant trois heures, cinq jours sur sept; et leurs équipes se classaient parmi les cinq meilleures du championnat national.

Les cas (groupe expérimental) étaient composés de 63 joueurs consommant en moyenne cinq cigarettes par jour, depuis trois ans au moins. Les 63 autres (groupe témoin) étaient non fumeurs. Tous les sujets ont subi des examens cliniques, pulmonaire, cardiaque, et sanguins (goutte épaisse, numération formule sanguine). Tous ces sujets répondaient au critère d'inclusion suivant: absence de pathologies cardiorespiratoires et hématologiques patentes. Leurs caractéristiques anthropométriques sont rapportées dans le Tableau 1.

\section{Procédure expérimentale}

L'étude s'est déroulée à Brazzaville au cours du mois de février 2009, et à PointeNoire en mars 2009. Le niveau de dépendance nicotinique a été évalué par le biais du questionnaire de Fageström (1978). Par la suite, les paramètres anthropométriques suivantes ont été mesurés : la taille ( $\mathrm{T}$ en $\mathrm{cm})$, la masse corporelle ( $\mathrm{m}$ en $\mathrm{kg}$ ), la longueur du membre inférieur (LMI, en $\mathrm{cm}$ ), les périmètres de la cuisse et du mollet, les épaisseurs des plis cutanés de la cuisse et du mollet (à l'aide d'une pince Harpenden calibrée à $10 \mathrm{~g} / \mathrm{mm}^{2}$ ). De plus, le volume du membre inférieur (VMI) a été déterminé par la méthode des cônes tronqués de Jones et Pearson (1969), selon l'expression :

VMI $\left(\mathrm{en} \mathrm{cm}^{3}\right)=[(\mathrm{LMI})[\mathrm{SC}+\sqrt{ }(\mathrm{SC})(\mathrm{SM})+$ $\mathrm{SM}$ / 3 où LMI est la longueur du membre inférieur; SC et SM sont les surfaces des sections musculaires respectivement de la cuisse et du mollet, estimées par la formule (Heymsfield et al., 1982) : $\mathrm{S}=\left[(\mathrm{C}-\pi \mathrm{EP})^{2}-\right.$ $\mathrm{F}] / 4 \pi$ où $\mathrm{C}$ représente la circonférence $\mathrm{du}$ 
segment, et EP l'épaisseur du pli mesuré au niveau de la cuisse et du mollet. F est un facteur égal à 10 chez l'homme.

\section{Epreuves d'effort physique}

Chaque sujet a ensuite participé à deux séances d'évaluation de l'aptitude physique : une première séance pour évaluer la puissance anaérobie alactique maximale (P.A.A.M.), et une deuxième séance pour mesurer la puissance anaérobie lactique (P.A.L.). Un intervalle de 24 heures au moins séparait les deux séances afin d'éviter les effets cumulatifs de la fatigue et d'une récupération incomplète. Les sujets ne devaient pas pratiquer une activité physique intense au cours des trois jours précédents les tests. Le jour du test, tous les sujets ne devaient pas boire pendant le déroulement de l'épreuve d'effort, et les fumeurs étaient astreints à ne pas consommer du tabac deux heures avant l'expérimentation. Toutes les épreuves se déroulaient entre $8 \mathrm{~h} 30$ et $10 \mathrm{~h}$ du matin, dans une salle aérée et spacieuse, à température bien régulée $\left(\mathrm{T}=24^{\circ} \mathrm{C}\right)$.

\section{Protocole d'évaluation de la puissance anaérobie alactique}

L'épreuve de détente verticale (jumptest) a obéi à un protocole proche du test de Sargent (1921). Elle a permis de relever la donnée $\mathrm{h}$ de la détente. Sa valeur a été obtenue en ôtant la taille du sujet bras levé (abduction du bras de $180^{\circ}$ avec rotation de l'omoplate, mains et doigts tendus), à la hauteur maximale touchée avec le bout des doigts (bras en abduction complète) lors d'un saut.

Trois sauts ont été accomplis. Seule la meilleure performance a été retenue, puis intégrée dans le calcul de la PAAM.

Pour réaliser cette mesure, une règle étalonnée de $1,20 \mathrm{~m}$ à $3,50 \mathrm{~m}$ a été fixée verticalement sur un mur. Les sujets, bout des doigts « talqués », ont laissé une empreinte sur la règle. Toutes les mesures ont été immédiatement précédées d'une flexion des membres inférieurs $\left(30^{\circ}\right.$ à $\left.45^{\circ}\right)$. Ils ont été réalisés avec un appui bipodal et sans course d'élan. Le balancé des bras d'arrière en avant lors de l'impulsion a été autorisé. La PAAM a été calculée à partir de la relation :

$\mathrm{PAAM}=(\mathrm{mg} \sqrt{ } \mathrm{g} \times \sqrt{ } \mathrm{h}) / 2$, relation obtenue en utilisant la loi de chute libre des corps $\mathrm{h}=$ $\mathrm{gt}^{2} / 2$ et la formule $\mathrm{P}$ (puissance) $=[\mathrm{W}$ (travail)]/t (temps). La valeur de l'accélération de la pesanteur terrestre g étant égale à 9,7804 $\mathrm{m} / \mathrm{s}^{2}$ au niveau de Brazzaville, la puissance anaérobie alactique maximale, exprimée en watts, a été en définitive déterminée selon l'expression : PAAM $=21,61 \times \mathrm{m} \times \sqrt{ } \mathrm{h}$.

L'unité de h est le mètre.

\section{Procédure d'évaluation de la puissance anaérobie}

Il s'agissait du test de la capacité anaérobie de 30 secondes ou test de Wingate, sur ergocyclomètre de marque Ergocard 800 $\mathrm{S}$, à frein électromagnétique. Le test consiste en un effort maximal d'emblée et d'une durée de 30 secondes contre une charge imposée de $75 \mathrm{~g} / \mathrm{kg}$ de poids corporel (soit environ $5 \mathrm{~kg}$ pour un sujet de $70 \mathrm{~kg}$ ). Le sujet devait pédaler le plus vite possible, le chronomètre et le compteur électronique étant mis simultanément en marche. Le compte-tour électronique indique la vitesse moyenne toutes les 5 secondes, et un enregistreur peut lui être associé pour le tracé de la courbe de vitesse instantanée. Le travail total fourni pendant le test, exprimé en joules, représente l'ensemble de la capacité anaérobie alactique et lactique pour 30 secondes, à la charge imposée. Mais il est nécessaire de faire procéder à un échauffement préalable pendant 10 à 15 minutes sur l'ergocyclométre sans résistance (90 à 100 révolutions par minute), suivi d'un repos de 1 minute. L'épreuve était lancée, avec mise en jeu de la charge en 4 secondes. Le nombre de révolutions de pédalier était enregistré toutes les 5 secondes. Tout au long de l'épreuve, les sujets étaient encouragés. L'épreuve était arrêtée lorsque la vitesse n'était plus maintenue. La fréquence cardiaque était mesurée à l'aide d'un cardiofréquencemètre de type Sport-Tester et d'un palpeur fixé sur le lobe de l'oreille et relié à la console de l'ergocyclomètre. Dès la fin du 
test, le sujet était invité à pédaler à une fréquence libre contre une résistance très faible, afin d'éviter toute stase veineuse dans les membres inférieurs et un retour veineux déficient.

Des échantillons de sang ont été prélevés ( $5 \mu 1$ environ) avant, à la fin d'effort, et à $\mathrm{t}=2,5,10,15$ et 20 minutes pendant la phase de récupération, le sujet récupérant assis sur le cycloergomètre. Les prélèvements sanguins étaient effectués au cours et au décours de l'épreuve de pédalage. Un cathéter très fin et flexible était introduit dans une veine du pli du coude, pour éviter de piquer les sujets pendant sept fois. Un échantillon sanguin a été prélevé quinze minutes avant l'exercice pour déterminer les valeurs basales. Les jeux de seringues et tubes capillaires ont été préalablement héparinés pour recevoir les prélèvements. Le dosage des lactates a été réalisé par méthode enzymatique, à l'aide de l'appareil de mesure de lactates LACTATE PRO (Nikkon Medical Co, Japon).

\section{Variables étudiées}

Les puissances anaérobies alactique et lactique absolues, rapportées au poids corporel et au volume musculaire des membres inférieurs, ont constitué les premières variables de l'étude. Les autres variables concernaient les concentrations de lactates sanguins au repos, en fin d'effort et à 10 minutes au décours de l'épreuve cycloergométrique. Par ailleurs, les variations enregistrées ont été analysées chez les fumeurs en fonction du niveau de dépendance nicotinique.

\section{Analyse statistique}

Les résultats ont été exprimés sous forme de moyenne et erreur standard de la moyenne $(x \pm s)$. Pour chaque mesure, les variations des différents paramètres enregistrés, à l'effort et au cours de la récupération, entre fumeurs $(F)$ et non fumeurs (NF) ont été comparées à l'aide du test non paramétrique U de Mann-Whitney, conformément aux recommandations de Stuart (1985). En effet, les variables hématologiques ont souvent une répartition non gaussienne. Les écarts observés chez les fumeurs des différents niveaux de dépendance nicotinique ont été examinés par le test $a$ posteriori de Scheffé après une analyse de variance (ANOVA). Le seuil de significativité statistique a été fixé à $\mathrm{p}<005$, avec pour logiciel de traitement des données Stata $^{\circledR}$ version 12.0.

\section{RESULTATS}

Les valeurs des puissances anaérobies alactique maximales (PAAM), rapportées au volume musculaire, des fumeurs étaient significativement $(\mathrm{p}<0,001)$ plus faibles que celles enregistrées chez les non fumeurs (Tableau 2). Par contre, aucune différence significative des valeurs de la PAAM rapportées aux poids corporel n'a été observée entre les deux groupes. Concernant les puissances anaérobies lactiques (PAL), le niveau de développement était statistiquement similaire entre les deux groupes (Tableau 2). Cependant, l'analyse de variance montre l'influence du degré d'intoxication tabagique sur les différentes variables bioénergétiques étudiées (Tableau 3). L'effet le plus significatif se retrouvait au niveau de la PAAM absolue $[\mathrm{F}(3,122)=62,87 ; \mathrm{p}<0,001]$, la PAAM rapportée au volume musculaire $[\mathrm{F}(3,122)=56,45 ; \mathrm{p}<0,001]$ et la PAL absolue $[\mathrm{F}(3,122)=35,95 ; \mathrm{p}<0,001]$. Les valeurs inférieures étaient retrouvées au niveau de la PAAM et la PAL rapportées au poids corporel : $\mathrm{F}(3,122)=3,04(\mathrm{p}>0,05)$ et $F(3,122)=4,36(p>0,05)$.

Par ailleurs, les concentrations moyennes de lactates sanguins, au repos et à la récupération, s'étaient plus élevées $(\mathrm{p}<0,001)$ chez les $\mathrm{F}$ par rapport à celles des NF (Tableau 4). Enfin, l'effet de la variable "dépendance nicotinique" sur les taux plasmatiques d'acide lactique (Tableau 5 et Figure1) était également significatif $(\mathrm{p}<0,001)$. 
Tableau 1 : Caractéristiques anthropométriques des sujets et différences entre fumeurs et non Fumeurs.

\begin{tabular}{|c|c|c|c|}
\hline Paramètres & $\begin{array}{c}\text { Fumeurs } \\
(\mathrm{n}=63)\end{array}$ & $\begin{array}{l}\text { Non fumeurs } \\
\quad(n=63)\end{array}$ & $\mathbf{p}$ \\
\hline Age (ans) & $24,6 \pm 2,9$ & $24,9 \pm 3,8$ & NS \\
\hline Taille (cm) & $172,4 \pm 6,8$ & $173,6 \pm 4,7$ & NS \\
\hline Poids (kg) & $64,1 \pm 5,8$ & $68,3 \pm 3,1^{* * * *}$ & $<0,001$ \\
\hline $\operatorname{IMC~}\left(\mathrm{kg} \mathrm{/m^{2 }}\right)$ & $21,2 \pm 1,8$ & $22,8 \pm 0,7^{* * * *}$ & $<0,001$ \\
\hline VMI $\left(\mathbf{d m}^{3}\right)$ & $8,6 \pm 1,3$ & $9,7 \pm 2,1 * * *$ & $<0,001$ \\
\hline \multirow{2}{*}{\multicolumn{4}{|c|}{ 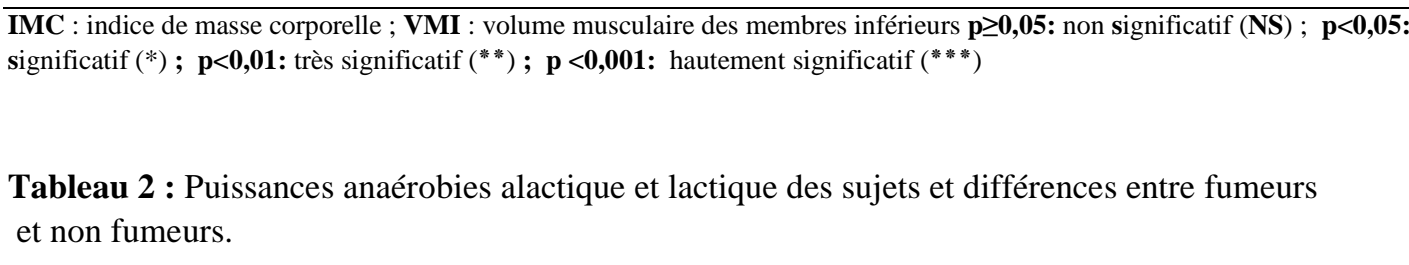 }} \\
\hline & & & \\
\hline Paramètres & $\begin{array}{l}\text { Fumeurs } \\
(\mathrm{n}=63)\end{array}$ & $\begin{array}{l}\text { Non fumeurs } \\
\quad(n=63)\end{array}$ & $\mathbf{p}$ \\
\hline PAAM (w) & $942 \pm 81,4$ & $995,2 \pm 60,3$ **** & $<0,001$ \\
\hline PAAM (w/kg) & $14,7 \pm 3,1$ & $14,5 \pm 4,3$ & NS \\
\hline PAAM (w/dm $\left.{ }^{3}\right)$ & $109,5 \pm 11,2^{* *}$ & $102,5 \pm 9,4$ & $<0,01$ \\
\hline PAL (w) & $281,6 \pm 24,7$ & $326,3 \pm 31,3^{* * * *}$ & $<0,001$ \\
\hline PAL (w/kg) & $4,4 \pm 2,1$ & $4,8 \pm 1,6$ & NS \\
\hline PAL (w/dm $\left.{ }^{3}\right)$ & $32,7 \pm 4,8$ & $33,6 \pm 3,1$ & NS \\
\hline
\end{tabular}

PAAM: puissance anaérobie alactique maximale ; PAL: puissance anaérobie lactique maximale $\mathbf{p} \geq \mathbf{0 , 0 5}$ : non significatif (NS) ; $\mathbf{p}<\mathbf{0 , 0 5}$ : significatif $(*) ; \mathbf{p}<\mathbf{0 , 0 1 :}$ très significatif $\left({ }^{* *}\right) ; \mathbf{p}<\mathbf{0 , 0 0 1}$ : hautement significatif $(* * *)$ 
Tableau 3 : Puissances anaérobies alactique et lactique en fonction du niveau de dépendance tabagique. Analyse de variance (p).

\begin{tabular}{|c|c|c|c|c|c|}
\hline Paramètres & $\begin{array}{c}\text { Niveau } 1 \\
(n=22)\end{array}$ & $\begin{array}{c}\text { Niveau } 2 \\
(n=17)\end{array}$ & $\begin{array}{r}\text { Niveau } 3 \\
(n=14)\end{array}$ & $\begin{array}{r}\text { Niveau } 4 \\
(n=10)\end{array}$ & $\mathbf{p}$ \\
\hline PAAM (w) & $1037,4 \pm 112,5 * * *$ & $978,5 \pm 73,6$ & $877,8 \pm 69,3$ & $806,2 \pm 71,4$ & $<0,001$ \\
\hline PAAM (w/kg) & $16,1 \pm 5,2^{*}$ & $15,5 \pm 3,8$ & $14,0 \pm 4,1$ & $13,2 \pm 3,3$ & $<0,05$ \\
\hline PAAM (w/dm $\left.{ }^{3}\right)$ & $125,3 \pm 8,4 * * *$ & $113,6 \pm 10,2$ & $101,5 \pm 12,1$ & $97,6 \pm 9,3$ & $<0,001$ \\
\hline PAL (w) & $311,7 \pm 18,2^{* * * *}$ & $293,8 \pm 22,3$ & $270,1 \pm 17,4$ & $248,8 \pm 19,6$ & $<0,001$ \\
\hline PAL (w/kg) & $5,3 \pm 1,8^{* *}$ & $4,7 \pm 2,3$ & $4,3 \pm 1,9$ & $4,1 \pm 2,1$ & $<0,01$ \\
\hline PAL (w/kg) & $35,2 \pm 3,5^{* * * *}$ & $33,4 \pm 4,2$ & $31,9 \pm 3,9$ & $30,3 \pm 4,3$ & $<0,001$ \\
\hline
\end{tabular}

PAAM: Puissance anaérobie alactique maximale ; PAL: Puissance anaérobie lactique maximale ; $\mathbf{p} \geq \mathbf{0 , 0 5}$ : non significatif (NS) ; $\mathbf{p}<\mathbf{0 , 0 5}$ : significatif $(*) ; \mathbf{p}<\mathbf{0 , 0 1}$ : très significatif $\left({ }^{* *}\right) ; \mathbf{p}<\mathbf{0 , 0 0 1}$ : hautement significatif $\left({ }^{* * *}\right)$

Tableau 4 : Résultats comparés de la lactatémie des sujets fumeurs et non fumeurs.

\begin{tabular}{|c|c|c|c|}
\hline Paramètres & $\begin{array}{c}\text { Fumeurs } \\
(n=63)\end{array}$ & $\begin{array}{l}\text { Non fumeurs } \\
\quad(n=63)\end{array}$ & $\mathbf{p}$ \\
\hline $\mathbf{L} \mathbf{A}_{\mathrm{a}, \mathrm{e}}(\mathbf{m m o l} / \mathbf{L})$ & $1,73 \pm 0,22^{* * *}$ & $1,24 \pm 0,18$ & $<0,001$ \\
\hline $\mathbf{L} \mathbf{A}_{\mathrm{f}, \mathrm{e}}(\mathbf{m m o l} / \mathbf{L})$ & $14,34 \pm 2,05^{* * *}$ & $11,18 \pm 1,99$ & $<0,001$ \\
\hline $\mathbf{L ~} A_{a, r}(\mathbf{m m o l} / L)$ & $2,12 \pm 0,53^{* * *}$ & $1,17 \pm 0,23$ & $<0,001$ \\
\hline
\end{tabular}


Tableau 5 : Comparaison de la lactatémie en fonction du niveau de dépendance tabagique. Analyse de variance (p).

\begin{tabular}{lccccc}
\hline Paramètres & $\begin{array}{c}\text { Niveau 1 } \\
(\mathbf{n = 2 2})\end{array}$ & $\begin{array}{c}\text { Niveau 2 } \\
(\mathbf{n = 1 7})\end{array}$ & $\begin{array}{c}\text { Niveau 3 } \\
(\mathbf{n = 1 4})\end{array}$ & $\begin{array}{c}\text { Niveau 4 } \\
(\mathbf{n = 1 0})\end{array}$ & $\mathbf{p}$ \\
\hline $\mathbf{L A}_{\mathbf{a}, \mathbf{e}}(\mathbf{m m o l} / \mathbf{L})$ & $1,64 \pm 0,15$ & $1,70 \pm 0,24$ & $1,77 \pm 0,18$ & $1,81 \pm 0,27$ & $<0,01$ \\
& & & & & NS \\
$\mathbf{L ~} \mathbf{A}_{\mathbf{f}, \mathbf{e}}(\mathbf{m m o l} / \mathbf{L})$ & $13,83 \pm 1,81$ & $14,31 \pm 2,03$ & $14,64 \pm 1,75$ & $14,64 \pm 1,75$ & $<0,001$ \\
& & & & & \\
\hline
\end{tabular}

Lactatémie : $\mathbf{L} \mathbf{A}_{\mathrm{a}, \mathrm{e}}$ avant effort ; $\mathbf{L} \mathbf{A}_{\mathbf{f , e}}$ : en fin d'effort $; \mathbf{L ~} \mathbf{A}_{\mathrm{a}, \mathrm{r}}$ : après 10 minutes de repos $\mathbf{p} \geq \mathbf{0 , 0 5}$ : non significatif (NS) ; $\mathbf{p}<\mathbf{0 , 0 5}$ : significatif $(*) ; \mathbf{p}<\mathbf{0 , 0 1}$ : très significatif $(* *) ; \mathbf{p}<\mathbf{0 , 0 0 1}$ : hautement significatif $\left({ }^{* * *}\right)$

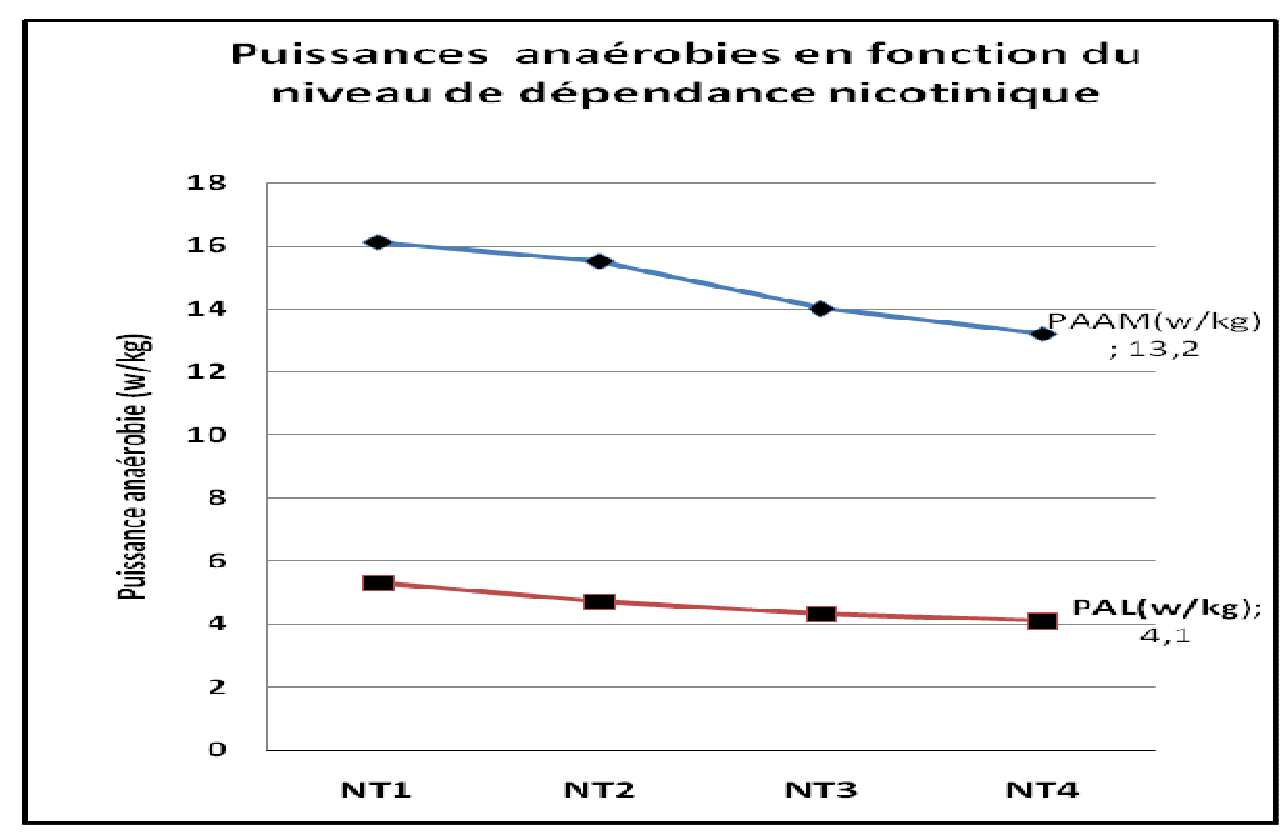

Figure 1: Résultats comparés des puissances anaérobies alactique et lactique en fonction du niveau de dépendance tabagique

PAAM: Puissance anaérobie alactique maximale

PAL : Puissance anaérobie lactique 


\section{DISCUSSION}

Puissance anaérobie alactique maximale

Les valeurs des puissances anaérobies maximales absolues et rapportées au volume musculaire, développées par les fumeurs, se sont avérées significativement $(p<0,01)$ plus faibles par rapport à celles des non fumeurs (Tableau 2). Les écarts maximaux représentent respectivement $13,3 \%$ et $15,2 \%$. Nos résultats sont superposables à ceux de Carvalho et al. (2010) qui ont retrouvé, au terme d'une épreuve cycloergométrique, des différences significatives de la puissance musculaire explosive entre $\mathrm{F}$ et NF. Cependant, les performances relevées étaient inférieures à celles de notre série : 885,3 \pm $61,8 \mathrm{w}$ versus $942,1 \pm 81,4 \mathrm{w}$. Les PAAM plus élevées, enregistrées dans notre étude, pourraient donc s'expliquer partiellement par une exécution du test dans une condition qui est plus proche de la situation du jeu pendant les rencontres de football (Vandewalle et Friemel, 1999). En effet, lors d'une épreuve cycloergométrique, le corps est porté (sujet assis sur la selle) et les membres inférieurs interviennent de façon alternée, alors que pendant le test de détente verticale sans élan ce sont les jambes qui supportent tout le poids $\mathrm{du}$ corps. De plus, nos résultats sont en concordance avec ceux rapportés par d'autres études: des PAAM élevées ont été obtenues chez des sujets de race noire au terme de l'épreuve de détente verticale (Bongbele et al., 1999 ; Bouchard et al., 2007).

Les différences observées entre les fumeurs et les non fumeurs sont imputables aux modifications métaboliques et anatomiques, résultant de l'action conjuguée de la nicotine et du monoxyde de carbone (CO). En effet, il est bien établi que la nicotine provoque la libération accrue des catécholamines et notamment de l'adrénaline, processus induisant une diminution des réserves d'énergie mobilisables immédiatement (créatine phosphate), une augmentation de la lactatogenèse musculaire, et l'hyper-lactatémie du milieu intérieur après la mise en action des muscles (Walsh et al.,
2000). Par ailleurs, d'autres auteurs rapportent que cette libération hormonale entraîne également une diminution de la masse corporelle totale, notamment de la masse musculaire susceptible de produire une plus grande force lors des épreuves fonctionnelles telles que le saut vertical. (Flaudes et Jimenes, 1991 ; Packa Tchissambou et al., 2001). Cette hypothèse est en accord avec les différences significatives $(p<0,01)$ des puissances rapportées au volume musculaire, entre les fumeurs et non fumeurs (Tableau 2). Par ailleurs, le monoxyde de carbone de la fumée de tabac diminue le pouvoir oxyphorique du sang (Turino, 2001) et entraîne l'hypoxie musculaire (Celsing et al., 2001) ; ce qui accroît encore la production de l'acide lactique musculaire et la lactatémie du milieu intérieur au cours de l'exercice. Carvalho et al. (2010) ont récemment montré que la désoxygénation du muscle chez le fumeur s'installe même pour des charges de travail situées au-dessous du seuil d'acidose lactique. Toutefois, une procédure minutieuse d'étude des effets du CO devrait associer une détermination de la carboxyhémoglobinémie, laquelle n'a pu être effectuée en raison de la faiblesse du plateau technique (absence des techniques de détection gazométrique, chromatographique, et spectrophotométrique); ce qui constitue une faiblesse de l'étude. En effet, la connaissance de la concentration du $\mathrm{HbCO}$, de sa vitesse de formation et d'élimination, concourt à mieux expliquer les processus de transport et de diffusion entre les cellules et les tissus, ainsi que les effets intracellulaires induits. Enfin, les résultats de ce travail montrent une limitation de la puissance anaérobie alactique, liée au degré d'intoxication tabagique (Tableau 3 et Figure 1). Cette décroissance est également observée chez des sportifs par Klausen et al. (2003), Stuart et al. (2004), avec des pertes voisines de $27 \%$, supérieures par rapport à la nôtre, $20 \%$. Cette supériorité, retrouvée par les auteurs occidentaux, est en relation avec la prévalence du tabagisme dans ces pays. L'augmentation du nombre de paquets de 
cigarettes induit donc une élévation significative de la nicotinémie et de l'hypoxie tissulaire.

\section{Puissance anaérobie lactique}

Les résultats présentés dans le Tableau 2 indiquent que les puissances anaérobies lactiques absolues des fumeurs sont significativement $(\mathrm{p}<0,001)$ inférieures à celles des non fumeurs. Ces différences peuvent être discutées en termes de limitation plus accentuée de la glycolyse au niveau de la fibre musculaire des footballeurs fumeurs au cours de l'exercice et à leur faible masse musculaire (Tableau 1). En effet, l'action cholinergique de la nicotine et hypoxique du monoxyde de carbone provoque une élévation notoire de la production de l'acide lactique et un abaissement du pH. Or, l'acidification des fibres musculaires diminue la capacité des fibres à libérer le calcium, avec pour conséquence la réduction de leur contractilité (Marini, 1987). Cependant, des dosages de la nicotinémie par le biais des biopsies auraient pu mieux objectiver ces hypothèses. Quant aux faibles valeurs des puissances anaérobies lactiques rapportées au volume musculaire, notées chez les fumeurs (Tableaux 1 et 2), elles pourraient être attribuées à la masse musculaire amoindrie des segments mesurés. Notre travail a également mis en évidence une diminution significative de la puissance anaérobie lactique avec l'augmentation de la dépendance tabagique. Cette relation est, à n'en point douter, à l'accent accru du cumul des actions de la nicotine et du CO.

Concernant les variations de la lactatémie, les taux plasmiques enregistrés chez les fumeurs au repos, en fin d'effort et à la récupération étaient significativement ( $\mathrm{p}<0,001$ ) plus élevés (Tableau 4).

Nos valeurs sont également supérieures à celles rapportées par Berthoin (2000), au cours d'exercices maximaux chez des sujets entraînés non fumeurs : 11,3 à $16 \mathrm{mmol} / \mathrm{L}$. De plus, l'analyse de variance indique un effet significatif de la dépendance nicotinique sur les concentrations de lactates sanguins
(Tableau 5 et Figure 1). La forte lactatémie enregistrée chez les fumeurs est imputable à l'action du CO inhalé, en diminuant nettement la capacité de sa diffusion et de son oxydation (IPCS, 1999). Toutefois, nos valeurs sont plus élevées que celles notées dans une étude antérieure chez les fumeurs (Packa Tchissambou et al., 2010): 5,18 $\pm 0,02$ $\mathrm{mmol} / \mathrm{L}$. Ces différences sont liées au protocole expérimental. En effet, dans l'étude suscitée, les mesures ont été réalisées après une épreuve d'effort sur tapis roulant. Or il a été montré que la concentration de lactates sanguins est plus importante lors d'un effort réalisé sur ergocyclomètre que lors d'un effort de même intensité sur tapis roulant (Berthoin et al., 2000). En effet, la lactatémie dépend de la masse de muscle squelettique mise en jeu au cours d'un exercice ; elle est d'autant plus élevée que cette masse est plus faible (Boulay et al., 1985). Ainsi, chez les fumeurs, à l'hypoxie locale des muscles au travail, s'ajoute l'hypoxie tissulaire due au tabac. Ceci concourt à la forte augmentation de la lactatémie. Enfin, au cours de notre étude les taux de lactates sanguins ont diminué de $85,2 \%$ entre la fin de l'effort et la $10^{\mathrm{e}}$ minute de récupération. Cette élimination moins rapide de l'acide lactique est imputable sans nul doute à la limitation de l'oxydation des lactates par le foie, le rein, le myocarde et le tissu musculaire lui-même.

\section{Conclusion}

Nos résultats, tout en comportant certaines faiblesses, valident l'hypothèse selon laquelle le tabagisme a des effets délétères sur le métabolisme anaérobie. De plus, la lactatémie est moins importante chez les petits fumeurs, contrairement aux grands fumeurs. La diminution de la puissance musculaire repose sur l'hypoxie tissulaire, les modifications des activités enzymatiques au niveau musculaire et la répartition des fibres des muscles en action. Toutefois, des travaux en cours, croisant systématiquement plusieurs variables physiologiques, pourront mieux 
éclairer les mécanismes intracellulaires de toxicité du tabac.

\section{REFERENCES}

Bar-Or O, Dotan R, Inbar O, Rothstein A, Karlsson J, Tesch P. 1980. Anaerobic capacity and muscle fiber type distribution in man. Int. J. Sports Med., 1: 82-5.

Batra V, Paktar AA, Berrettini WH, Weinstein SP, Leone FT. 2003. The genetics determinants of smoking. Chest, 123(5): 1730-1739.

Berthoin S, Pelayo P, Baquet G, Marais G, Robin H. 2000. Effets des variations du volume plasmatique sur les concentrations de lactate et leur cinétique de récupération après des exercices maximaux et submaximaux. Science \& Sports, 15: 31-39.

Bongbele, Massamba A, Mboussa A, Kiori R. 1999. Influence du test d'évaluation sur la puissance anaérobie maximale chez des joueurs d'élite mélano-africains. STAPS, 49: 99-106.

Booth FW, Thomason DH. 1991. Molecular and cellular adaptations of muscle in response to exercise: perspectives of various models. Physiol. Rev., 71: 541585.

Boulay MR, Lortie G, Simoneau JA, Hamen P, Leblanc C, Bouchard C. 1985. Specificity of aerobic and anaerobic work capacities and power. Int. J. Sports Med., 6: 325-328.

Calfee R, Padal P. 2006. Popular ergogenic drugs and supplements in young athletes.

Pediatrics, 117(3): 577-589.

Celsing F, Svedenhay J, Pihlsted P, Ekblöm B. 2001. Effects of tobacco consumption on maximal anaerobic power in individuals with high and low nicotine concentrations. Acta. Physiol. Scand, 143: 1147-54.

Fageström KO. 1978. Measuring degree of physical dependence to tobacco smoking with reference to individualization of treatment. Addict Beth, 3: 235-41.
Groussard C, Rouger K, Gratas-Delamarche A, Morel I, Chevagne M, Cillard J, Delamarche P. 1999. Effet du lactate sur la production de radicaux libres: étude in vitro. Science \& Sports, 14: 149-152.

Heymsfield SM, Mc Manus C, Smith J, Stevins V, Nixon D W. 1982. Anthropometric measurement of mass: revised equations for calculating bonefree arm muscle area. Am. J. Clin. Nutr., 7: 680-690.

IPCS. 1999. Carbon monoxide. In Environmental Health Criteria, ( $2^{\text {nd }}$ edn $)$. JPCS/WHO: Geneva; 464 p.

Jones PRM, Pearson J. 1969. Anthropometric determination of leg fat and muscle plus bone volumes in young males and female adult. J. Physiol., 204: 63-6.

Karlsson J. 1971. Lactate and phosphagen concentrations in working muscle of man with special reference to oxygen deficit at the onset of work. Acta. Physiol. Scand, 358: $1-72$.

Klausen K, Andersen C, Nandrup S. 2003. Acute effects of cigarette smoking and inhalation of carbon monoxide during maximal exercise. Eur. J. Appl. Physiol., 71: 1371-1379.

Lanston P, Gorman D, Runchman W, Upton R. 2006. The effects of nicotinemia on cycling AMP in skeletal muscles. Toxicology, 128: 1723-32.

Longo LD. 2006. The biological effects of carbon monoxide and nicotinemia on skeletal fiber types and enzymes activities in humans. Science, 224: 2521-2529.

Lormes W, Lehmann M, Steinacker JM. 2007. The problem to study relations between plasma lactate and fiber composition. In Physiology of Exercise, Amstrong N, Kirby RJ (eds). E \& FN Spon: London; 405-411.

Maehara K, Riley M, Galassetti P, Barstow TJ, Wasserman K. 1997. Effect of hypoxia and carbon monoxide on muscle oxygenation during exercise. Am. J. Respir. Crit. Care Med., 155: 229-35. 
Marini JF. 1987. L'étude des adaptations métaboliques du muscle squelettique : effets de l'entraînement sur les activités enzymatiques. Science et Motricité, 1: 3644.

Oyono-Enguelle S, Freund H, Lampert E, Lonsdorfer A, Lonsdorfer J. 1993. Modelling lactate kinetics during recovery from muscular exercise in humans. I. Influence of some physiological factors. Science \& Sports, 3: $181-187$

Packa TB, Massamba A, Mabiala Babela JR, Kissambou Mouanou J, Mboussa J, Senga P. 2004. Effets du tabagisme et du niveau de dépendance nicotinique sur la capacité aérobie chez le sportif. Rev. Mal. Respir., 21: 59-66.

Packa TB, Massamba A, Mabiala Babela JR, Senga P, Gbeassor M. 2010. Variations de la lactatémie à l'effort et au cours de la récupération chez des sportifs fumeurs. Ann. Afr. Med., 3(4): 560-565.

Packa TB, Massamba A, Oniangue R, Kissambou Mouanou J, Makanga M, Senga P. 2001. Effet du tabagisme sur le poids corporel et les capacités cardiorespiratoires chez les sportifs congolais. Cahiers Santé, 11(2): 161-166.

Packa TB. 2003. Effets du tabagisme sur la composition corporelle, l'aptitude physique et les adaptations hémorespiratoires. Thèse de doctorat d'état en physiologie animale, Université Marien NGOUABI, Brazzaville, p.351.

Packa TB, Massamba A. 2009. Etude épidémiologique du tabagisme en milieu sportif. Rapport de recherche, Ministère de la Santé / Ministère des Sports, Brazzaville, Congo, 52p.
Perusse L. 2001. Les bases génétiques et moléculaires de la performance et de l'adaptation à l'exercice. Science \& Sports, 16: 1986-195.

Sargent DA. 1921. The physical test of a man. Am. Phys. Educ. Rev., 26:188-194.

Stewart RD, Baretta ED, Platte LR, Stewart EB, Kalbfleisch JH. 2004. Fitness and carbohemoglobin levels in American athletes blood donors. J. Am. Med. Assoc., 272: 2687-2695.

Stuart J. 1985. Design principles for clinical and laboratory studies of erythrocyte deformability and lactataemia. Clin. Hemorheol, 5:59-69.

Tesch P. 1976. Muscle fatigue in man with special reference to lactate accumulation during short term intense exercise. Acta. Physiol. Scand, 480-4.

Thorstenson A, Grimby G, Karlsson J. 1976. Force-velocity relations and fiber composition in human knee extensor muscles. J. Appl. Physiol., 40: 12-16.

Turino GM. 2001. Effect of carbon monoxide on the muscular and cardiorespiratory system. Carbon monoxide: physiology and biochemistry. Circulation, 103: 753A-759A.

Walsh MM, Ellison J, Rilton JF, Chesney M, Ernster VL. 2000. Spit (Smokeless) tobacco use by high school baseball athletes in California. Tob. Control, 9: 1132-1139.

Wagner JA, Horvath SM, Andrew GM, Gottle WH, Bedi JF. 2000. Lactate concentrations, smoking history, and exercise. Am. Hum. Biol., 27(5): 799-811. 